\title{
Comparación de la castración quirúrgica al nacimiento versus inmunocastration sobre las características de la canal y carne en machos Holstein
}

Jorge A. Cervantes-Cazares ${ }^{\text {a }}$

Cristina Pérez-Linares ${ }^{a *}$

Fernando Figueroa-Saavedra $^{\text {a }}$

Alma R. Tamayo-Sosa ${ }^{a}$

Alberto Barreras-Serrano ${ }^{a}$

Francisco G. Ríos-Rincón ${ }^{b}$

Eduardo Sánchez-López ${ }^{\text {a }}$

Issa C. García-Reynoso ${ }^{\text {a }}$

Pedro Mendoza Peraza ${ }^{\text {a }}$

Angelina León Villanueva ${ }^{a}$

Luis A. García-Vega ${ }^{c}$

a Universidad Autónoma de Baja California, Instituto de Investigaciones en Ciencias Veterinarias, A. Obregón y J. Carrillo s/n Col. Nueva, Mexicali, Baja California, 21100, México.

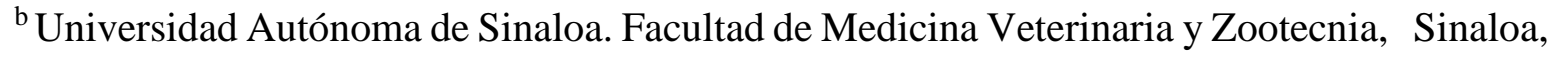
México.

${ }^{\mathrm{c}}$ Ganadera Mexicali, S.A. de C.V, Baja California, México.

*Autor de correspondencia: cristinapl@yahoo.com 


\section{Resumen:}

El objetivo fue comparar el efecto de la castración quirúrgica al nacimiento vs immunocastración, sobre las características de la canal y carne en machos Holstein en engorda; se utilizaron 720 machos Holstein aproximadamente de 7 a 8 meses de edad con peso inicial de $240.82 \mathrm{~kg}$. Se formaron 2 tratamientos con 4 corrales de 90 animales en cada uno: toros castrados quirúrgicamente que fueron castrados $24 \mathrm{~h}$ después del nacimiento y toros inmunocastrados vacunados con Bopriva aplicando cuatro dosis, al día 1, 21, 101 y 181 de engorda. Se tomaron pesos individuales en cada vacunación. Los animales se sacrificaron a los 242 días de engorda. A partir de la segunda vacunación se observaron diferencias $(P<0.05)$ en pesos, presentando valores mas altos los animales castrados quirúrgicamente. El peso final al sacrificio, peso de la canal caliente, peso de la canal fría, espesor de grasa dorsal y área del ojo de la costilla fueron diferentes $(P<0.05)$ entre tratamientos, observando valores más altos en los machos castrados quirúrgicamente. En la carne, el pH y EC fueron similares $(P>0.05)$ entre tratamientos mientras que los valores de $\mathrm{b}^{*}, \mathrm{C}^{*} \mathrm{y} \mathrm{H}^{*}$ fueron más altos $(P<0.05)$ en los animales inmunocastrados. Para fines de producción, el sacrificar los machos Holstein al nacimiento, se obtienen animales más pesados y con mejores características en la canal; sin embargo es importante evaluar el impacto del bienestar animal por la castración al nacimiento.

Palabras clave: Inmunocastración, Machos Holstein, Evaluación de la canal.

Recibido: 07/05/2018

Aceptado: 11/04/2019

\section{Introducción}

En la producción del ganado de carne, la castración es frecuentemente utilizada como una herramienta de manejo que proporciona grandes ventajas, incluyendo una reducción en el comportamiento agresivo y sexual, lo que resulta en un fácil y seguro manejo, lo cual promueve una mejor calidad de las canales através del incremento de la deposición de grasa de cobertura y menos daño en las canales por efecto de montas o agresiones en el corral de finalización, influyendo favorablemente en bienestar animal ${ }^{(1-5)}$. Sin embargo, la castración quirurgica requiere un trabajo y costo adicional, y provoca dolor prolongado en el animal ${ }^{(6,7)}$, infecciones y sangrados ${ }^{(8)}$ y en algunos casos la muerte ${ }^{(9)}$. 
La inmunocastración es un método no quirúrgico enfocado a preservar el bienestar de los bovinos que ingresan al corral de finalización intensiva ${ }^{(10)}$; mediante este procedimiento, los machos inmunocastrados producen un anticuerpo contra $\mathrm{GnRh}$ y en consecuencia reduce las concentraciones de testosterona $^{(11)}$ y disminuye la actividad física ${ }^{(12)}$. En años recientes, en los corrales de engorda se han incluido a machos de la raza Holstein como una alternativa productiva; este grupo racial muestra características que lo hacen diferente de las razas productoras de carne tradicionales, ya que tiene un temperamento amigable y juguetón, pero pueden tornarse mayormente agresivos si se conservan como toros enteros ${ }^{(13)}$. El uso de la inmunocastración se ha probado en diferentes razas, con diferentes programas de vacunación, dietas y programas de implante ${ }^{(14-17)}$, sin embargo, es necesario evaluar la aplicación de programas de castración inmunológica bajo diferentes sistemas de producción (razas, dietas, implantes), en particular al ganado Holstein bajo un sistema de producción comercial donde se exige pesos finales mayores a $550 \mathrm{~kg}$ para su sacrificio. Por lo anterior el objetivo de este estudio fue comparar el efecto de la castración quirúrgica al nacimiento vs. immunocastración sobre las características de la canal y carne en machos Holstein en engorda.

\section{Material y métodos}

\section{Localización geográfica}

El estudio se llevó a cabo en la ciudad de Mexicali, Baja California, el cual se encuentra ubicada a una $32^{\circ} 32^{\prime} 00 \mathrm{~N}, 115^{\circ} 12^{\prime} 41 \mathrm{O}$. La región está caracterizada por un clima seco desértico con una temperatura media de $34.7^{\circ} \mathrm{C}\left(-5^{\circ} \mathrm{C}\right.$ invierno y $50{ }^{\circ} \mathrm{C}$ verano), con una precipitación pluvial de $37 \mathrm{~mm}$, y una humedad relativa de acerca del $50 \%{ }^{(18)}$.

\section{Diseño del estudio}

Se utilizaron 720 machos Holstein de un mismo origen, con una edad de entre 7 y 8 meses a la llegada a la engorda y un peso vivo promedio de $240 \mathrm{~kg}$. Para el estudio se consideraron dos tratamientos: machos castrados quirúrgicamente (T1) y machos inmunocastrados (T2), donde fueron asignados aleatoriamente en cada tratamientos ubicando 90 animales por corral con cuatro corrales por tratamiento. Los animales se castraron a las $24 \mathrm{~h}$ después del nacimiento en los corrales del establo lechero.

\section{Manejo del ganado a la recepción de la engorda}

A las $24 \mathrm{~h}$ de la recepción de los animales, todos los animales fueron vacunados, desparasitados, y se aplicó un implante (un producto a base de acetato de trenbolona, estradiol 
y tylosina). A los animales inmunocastrados se les aplicó Bopriva® (Laboratorios Zoetis, Salud Animal, México) administrando $1 \mathrm{ml}$ subcutáneamente en cuatro ocasiones: (24 h después de la recepción y en los días 21, 101 y 181 del experimento), mientras que a los animales castrados quirúrgicamente se les administró $1 \mathrm{ml}$ de solución salina en los mismos días.

Se registró el peso vivo de cada animal durante los días 1, 21, 101 y 181 del experimento y antes del sacrificio. Los animales se alimentaron dos veces al día, siguiendo un programa de seis dietas típicas de la region norte del país compuesta de heno de trigo, sudán, sebo, DDG (granos secos de distelería), y una premezcla de minerales.

\section{Niveles de testosterona sérica}

Se seleccionaron aleatoriamente a 10 animales por corral para medir los niveles de testosterona sérica y se identificaron con un arete adicional. Las muestras de sangre se tomaron en los mismos días de aplicación de Bopriva y una muestra más durante el sacrificio de los animales, en la estación de desangrado en la línea de producción en la planta de sacrificio.

Aproximadamente $5 \mathrm{ml}$ de sangre fue extraída de la vena coccígea; las muestras se centrifugaron a 3,500 rpm para obtener el suero, usando la centrifuga TRIAC (Clay Adams, Modelo 0200, New Jersey, U.S.A.), y almacenadas a $-20{ }^{\circ} \mathrm{C}$ hasta que fue medida la concentración de testosterona, la cual se determinó usando el kit ELISA Testosterona (Bovina) ELISA Kit (Abnova Corporation, Taipei City, Taiwan), de acuerdo a las instrucciones del fabricante.

\section{Sacrificio de los animales}

Los animales se sacrificaron a los 242 días de engorda, una vez que alcanzaron un peso promedio de $607.85 \pm 12.89 \mathrm{~kg}$. El día de sacrificio los animales se arrearon por un vaquero a caballo cerca de $1.5 \mathrm{~km}$ hasta la planta de sacrificio. Los animales se mantuvieron en los corrales de reposo con acceso a agua por $5 \mathrm{~h}$ aproximadamente. Los animales se sacrificaron en una planta de sacrifico Tipo Inspeccion Federal (TIF), siguiendo la metodología descrita en la Norma Oficial Mexicana NOM-033-SAG/ZOO-2014, "Métodos para dar muerte a los animales domésticos y silvestres". 


\section{Evaluación de la canales}

Las canales de ambos tratamientos se almacenaron a $2{ }^{\circ} \mathrm{C}$ por $24 \mathrm{~h}$ y se realizó el corte entre la 12..$^{\mathrm{a}}$ y $13 .^{\mathrm{a}}$ costilla para obtener los datos de las canales. En un total de 120 canales por tratamiento (grupo de machos castrados e inmunocastrados) fueron disponibles por la planta de sacrificio para ser considerados para el estudio de las variables en la canal. Se midió el peso de la canal caliente y peso de la canal fría, deposición de grasa dorsal, grasa pélvica renal y del corazón $(\mathrm{KPH})$, marmoleo, área del ojo de la costilla (AOC), pH y color ( $\mathrm{L}^{*}, \mathrm{a}^{*}$, $\left.\mathrm{b}^{*}, \mathrm{C}^{*} \mathrm{y} \mathrm{H}^{*}\right)$. La grasa dorsal fue medida en $\mathrm{mm}$ utilizando una regla métrica ${ }^{(19)}$. El área del ojo de la costilla se evaluó usando una plantilla de plástico de acuerdo al método sugerido por Iowa State University. La cantidad estimada de grasa pélvica, renal y del corazón (KPH) se determinó subjetivamente y expresada como un porcentaje del peso de la canal caliente (PCC), y el marmoleo (en escala de trazas, ligero, pequeño, modesto, moderado, ligeramente abundante y moderadamente abundante) ${ }^{(20)}$.

\section{Calidad de la carne}

A las $48 \mathrm{~h}$ postmortem, un total de 80 muestas de carne de aproximadamente $1,000 \mathrm{~g}$ se obtuvieron del músculo Longissimus dorsi, 10 muestras aleatorias por cada corral en ambos tratamientos. Las muestras fueron empacadas al vacío, refrigeradas y enviadas al Laboratorio de Calidad de Productos de Origen Animal del Instituto de Investigaciones en Ciencias Veterinarias de la Universidad Autónoma de Baja California. El pH se determinó usando un potenciometro (HANNAH INSTRUMENTS Inc. pH 101): los valores de color ( $\mathrm{L}^{*}, \mathrm{a}^{*}, \mathrm{~b}^{*}$, $\left.\mathrm{C}^{*}, \mathrm{H}^{*}\right)$ fueron medidos en la superficie del corte del músculo Longissimus dorsi usando un espectofotómetro MINOLTA CM-2002 (Minolta camera, Co., Ltd., Japan) con un componente especular íncluido (SCI), un iluminante $\mathrm{D}_{65}$, y un observador de $10^{\circ}$, donde $\mathrm{L}^{*}$ es el índice de luminosidad, $\mathrm{a}^{*}$ es la intensidad de color rojo y $\mathrm{b}^{*}$ es la intensidad de color amarillo. El esfuerzo al corte se obtuvo usando piezas de carne de previamente cocidas obtenidas en forma perpendicular a las fibras musculares através de un sacabocado de $1 \mathrm{~cm}^{2}$ de diametro, utilizando un texturómetro (Lloyd Instruments, England) equipado con cuchillas Warner-Bratzler. Todas las mediciones se hicieron por triplicado.

\section{Análisis estadístico}

El modelo lineal estadístico utilizado para analizar la variación total en el estudio fue:

$Y_{i j}=\mu+\tau_{i}+\xi_{i j}$ con $i=1,2$ y $j=1,2, \ldots, r$ 
Donde:

$\boldsymbol{Y}_{i j}$ corresponde a los valores de $\mathrm{pH}$, color y esfuerzo al corte registrados en la carne, como variables de respuesta;

$\boldsymbol{\mu}$ es la media general, $\tau_{i}$ es el efecto fijo del tratamiento (castrados $v s$ inmunocastrados);

$\xi_{i j}$ es el efecto aleatorio del residual $\left[\xi_{i j} \sim \mathrm{NI}\left(0, \sigma_{e}^{2}\right)\right]$

Cuando los tratamientos resultaron una fuente de variación significativa $(P \leq 0.05)$, se compararon los valores medios de los tratamientos utilizando el procedimiento de Tukey. El análisis se realizó utilizando el procedimiento GLM del paquete estadístico SAS $^{(21)}$. Para comparar los niveles medios de testosterona entre tratamientos, incluyendo su comportamiento en el tiempo (día 1, 21, 101,181 y al desangrado), se utilizó el modelo lineal mixto:

$\mathrm{Y}_{\mathrm{ijk}}=\mu+\tau_{\mathrm{i}}+\mathrm{A}_{\mathrm{k}(\mathrm{i})}+\mathrm{D}_{\mathrm{j}}+(\tau \mathrm{D})_{\mathrm{ij}}+\xi_{\mathrm{ijk}} \operatorname{con} \mathrm{i}=1,2 ; \mathrm{j}=1,2, \ldots, 5, \mathrm{yk}=1,2, \ldots, \mathrm{r}$

Donde:

$\boldsymbol{Y}_{i j k}$ es la concentración de testosterona del k-ésimo animal tomado en el j-ésimo tiempo y perteneciente al i-ésimo tratamiento, como variable de respuesta;

$\boldsymbol{\mu}$ es la media general, $\tau_{i}$ es el efecto fijo del tratamiento;

$\boldsymbol{A}_{\boldsymbol{k}(i)}$ es el efecto aleatorio del animal dentro de tratamiento $\left[A_{k(i)} \sim \mathrm{NI}\left(0, \sigma_{a}^{2}\right)\right]$

$\boldsymbol{D}_{\boldsymbol{j}}$ es el efecto fijo del tiempo, en días, $(\tau D)_{i j}$ es el efecto de interacción tratamiento $\times$ tiempo;

$\xi_{i j k}$ es el efecto aleatorio del residual $\left[\xi_{i j} \sim \mathrm{NI}\left(0, \sigma_{e}^{2}\right)\right]$

El análisis se efectuó empleando el procedimiento MIXED utilizando el enunciado REPEATED, del paquete estadístico SAS (SAS Inst. Inc., Cary, NC). Para el análisis de los registros repetidos, el cual incluyera las correlaciones entre los registros del mismo animal y las varianzas heterogéneas entre registros en el tiempo, se evaluaron las estructuras de covarianzas: no-estructurada (NE), simetría compuesta (SC) y autorregresiva de primer orden (AR1), a través de los criterios de Akaike y de Schwartz, seleccionando como la mejor aquella con los menores valores para estos dos indicadores. En este análisis la estructura de covarianza seleccionada fue la no-estructurada (NE). Cuando la interacción tratamiento $\mathrm{x}$ tiempo resultó una fuente de variación significativa $(P \leq 0.05)$, se compararon las medias 
mínimo cuadráticas entre tratamientos, por cada nivel del factor tiempo, utilizando el procedimiento Tukey-Kramer ${ }^{(22)}$.

\section{Resultados y discusión}

\section{Ganancia de peso de los animales:}

Los pesos promedios registrados durante los días de vacunación se muestran en el Cuadro 1. A partir de la aplicación de la segunda vacuna (día 21) se observaron diferencias significativas $(P<0.05)$ por tratamiento, presentando valores mas altos los machos castrados, observándose este patrón hasta el día del sacrificio.

Cuadro 1: Valores medios \pm error estándar (EE) de peso $(\mathrm{kg})$ de los animales por tratamiento en los días de vacunación hasta el sacrificio

\begin{tabular}{lllll}
\hline Día de engorda & Castrados & Immunocastrados & EE & P> t \\
\hline 1 & 243.25 & 238.39 & 2.50 & $0.052 \mathrm{NS}$ \\
21 & 278.30 & 269.70 & 2.49 & $0.006^{*}$ \\
101 & 394.94 & 379.53 & 2.51 & $0.001^{*}$ \\
181 & 520.80 & 509.52 & 2.54 & $0.001^{*}$ \\
Sacrificio & 620.74 & 594.95 & 6.90 & $0.002^{*}$ \\
\hline
\end{tabular}

Se han reportado diferencias $(P>0.05)$ en el peso vivo de los animales con mayor peso en los machos tratados con bopriva que los castrados quirúrgicamente durante los 280 días de engorda, cuando la castración fue realizada a los 91 días de engorda ${ }^{(10)}$. En otro estudio ${ }^{(9)}$ reportaron pesos similares $(P>0.05)$ al sacrificio entre machos castrados quirúrgicamente e inmunocastrados con bopriva, realizando la castración dentro del periodo de la primera y segunda aplicación de los machos tratados con bopriva (15, 16 y 17 días después de la vacunación). Sin embargo, en el presente estudio, los animales se castraron al nacimiento, por lo que el tiempo de recuperación por alguna infección y pérdidas de peso ocasionadas por la castración no tuvieron repercusión a los 7 meses de edad, tiempo en que llegaron los animales de los corrales del establo a los corrales de la engorda.

\section{Concentraciones de testosterona sérica}

Los resultados de concentración de testosterona para ambos tratamientos se detectaron en niveles por debajo de $1 \mathrm{ng} / \mathrm{ml}$ durante todos los días de vacunación hasta el final del periodo de engorda. Los resultados confirman el efecto que tiene la vacuna en suprimir la 
concentración de testosterona sérica en el ganado, similar a lo reportado en otras investigaciones $^{(4,9)}$. Algunos autores sugieren que a los 7 meses puede ser la edad óptima para la inmunización contra GnRH y generar la máxima producción de anticuerpos en machos Bos taurus ${ }^{(22)}$, como así fue llevado a cabo en este estudio.

\section{Calidad de la canal y carne}

En el Cuadro 2 se presentan los valores medios de las características de las canales por tratamiento. Los resultados de PCC y PCF fueron diferentes entre tratamientos $(P<0.05)$ observando valores mas altos en las canales de machos castrados, otros estudios han observado que el PCC fueron similares en las canales de ambos tratamientos ${ }^{(14)}$. El EGD y AOC fueron diferentes $(P<0.05)$ entre tratamientos, los valores más altos se observaron en las canales de machos castrados; mientras que el KPH fue similar $(P>0.05)$ entre tratamientos. En otros estudios se han reportado valores similares $(P>0.05)$ de EGD y AOC en canales de machos Nellore castrados e inmunocastrados ${ }^{(14)}$; mientras que los resultados de EGD del presente estudio han sido observados en machos castrados e inmunocastrados de animales de razas Nellore y Nellore x Angus ${ }^{(15)}$. Los valores de AOC se han reportado más bajos a los observados en este estudio ${ }^{(15)}$ (castrados: $81.06 \pm 1.78 \mathrm{~cm}^{2}$ vs inmunocastrados: $83.61 \pm 1.73 \mathrm{~cm}^{2}$ ); estas diferencias pudieron deberse al tipo de razas en los animales utilizadas en el estudio. Algunos autores afirman que el ganado Holstein son de una estructura corporal más grande y más largos, y tienen un periodo mayor de días de engorda, lo que le permite desarrollar canales de mayor tamaño que razas de ganado de carne ${ }^{(23)}$.

Cuadro 2: Valores medios \pm error estándar (EE) de las variables de calidad de la canal por tratamiento

\begin{tabular}{lcccl}
\hline Variable & Castrados & Immunocastrados & EE & P> f \\
\hline PCC, $\mathrm{kg}$ & $376.60^{\mathrm{a}}$ & $362.61^{\mathrm{b}}$ & 4.14 & 0.0009 \\
PCF, $\mathrm{kg}$ & $374.87^{\mathrm{a}}$ & $361.38^{\mathrm{b}}$ & 4.09 & 0.0011 \\
EGD, cm & $0.65^{\mathrm{a}}$ & $0.55^{\mathrm{b}}$ & 0.33 & 0.0042 \\
AOC, cm ${ }^{2}$ & $91.41^{\mathrm{a}}$ & $86.83^{\mathrm{b}}$ & 1.54 & 0.0048 \\
KPH, \% & $1.49^{\mathrm{a}}$ & $1.60^{\mathrm{a}}$ & 0.09 & 0.2473 \\
\hline
\end{tabular}

$\mathrm{PCC}=$ peso de la canal caliente; $\mathrm{PCF}=$ peso de la canal fría; $\mathrm{EGD}=$ espesor de grasa dorsal; $\mathrm{AOC}=$ área del ojo de la costilla; $\mathrm{KPH}=$ grasa pélvica, renal y del corazón.

${ }^{\mathrm{a}, \mathrm{b}}$ Literales diferentes en el mismo renglón son diferentes $(P<0.05)$.

Se han reportado valores similares $(P>0.05)$ en machos castrados e inmunocastrados de EGD y AOC en ganado Holstein $x \mathrm{Cebu}^{(24)}$ y en ganando Nellore ${ }^{(10,14)}$. En un estudio donde los animales se sacrificaron a un peso promedio mas bajo, $477 \mathrm{~kg}$ (castrados) y $486 \mathrm{~kg}$ (inmunocastrados) $^{(9)}$, no observaron diferencias $(P>0.05)$ en PCC, EGD y AOC en machos 
Holstein, mientras que en este estudio los animales fueron sacrificados a pesos arriba de 600 $\mathrm{kg}$ los machos castrados y $594 \mathrm{~kg}$ los machos inmunocastrados. Es importante destacar, que las diferencias reportadas en este estudio con respecto a las variables de calidad de la canal de otros estudios ${ }^{(10,14,15)}$ pueden deberse a que la castración quirúrgica en estos estudios se realizó días antes o dentro del tiempo de la aplicación con Bopriva y en el presente estudio la castración fue a las $24 \mathrm{~h}$ del nacimiento y, a los 7 meses de edad, tiempo de llegada a los corrales de engorda, ya estaban totalmente recuperados de las implicaciones de una castración quirúrgica.

El número de canales que fueron clasificadas por marmoleo según la categoría de clasificación son descritas en el Cuadro 3; las frecuencias por categorías fueron similares $(P>0.05)$ en ambos tratamientos. El mayor número de canales en ambos tratamientos se clasificaron en las categorías "ligero" y "pequeño" para marmoleo. Estos resultados son similares $(P>0.05)$ a los reportados en otras investigaciones ${ }^{(25)}$ entre canales de machos inmunocastrados y castrados; mientras que, en otro estudio no reportaron diferencias $(P>0.05)$ en el porcentaje de grasa intramuscular entre canales de machos Holstein castrados e inmunocastrados ${ }^{(9)}$.

Cuadro 3: Clasificación del marmoleo en las canales por tratamiento

\begin{tabular}{llll}
\hline & $\begin{array}{l}\text { Castrados } \\
(\mathbf{n = 1 2 6})\end{array}$ & $\begin{array}{l}\text { Immunocastrados } \\
(\mathbf{n = 1 2 6})\end{array}$ & $\mathbf{P r}>\mathbf{X}^{\mathbf{2}}$ \\
\hline Trazas & 3 & 0 & -- \\
Ligero & 67 & 66 & 0.9309 \\
Pequeño & 46 & 50 & 0.6831 \\
Modesto & 9 & 0 & -- \\
Moderado & 1 & 10 & -- \\
\hline
\end{tabular}

No se observaron diferencias $(P>0.05)$ entre tratamientos para las categorías Ligero y Pequeño en prueba de hipótesis para igualdad de proporciones.

En el Cuadro 4, se observan los valores de las características físico-químicas de la carne de ambos tratamientos, los valores de $\mathrm{pH}$ de la carne fueron similares $(P>0.05)$ en canales de machos castrados e inmunocastrados, observando valores similares a rangos de una carne con $\mathrm{pH}$ normal de 5.5 a $5.8^{(26)}$; estos resultados fueron también han sido reportados con $\mathrm{pH}$ de 5.57 a las 24 h en canales de ganado Holstein ${ }^{(27)}$. 
Cuadro 4: Valores medios \pm error estándar (EE) de las variables físico-químicas de la carne

\begin{tabular}{lllll}
\hline Variables & Castrados & Immunocastrados & $\pm \mathbf{E E}$ & $\boldsymbol{P}>\mathbf{f}$ \\
\hline $\mathrm{pH}$ & $5.54^{\mathrm{a}}$ & $5.56^{\mathrm{a}}$ & 0.04 & 0.7163 \\
$\mathrm{~L}^{*}$ & $32.14^{\mathrm{a}}$ & $32.74^{\mathrm{a}}$ & 0.43 & 0.1731 \\
$\mathrm{a}^{*}$ & $11.97^{\mathrm{a}}$ & $10.70^{\mathrm{b}}$ & 0.33 & 0.0002 \\
$\mathrm{~b}^{*}$ & $7.93^{\mathrm{b}}$ & $12.86^{\mathrm{a}}$ & 0.47 & 0.0001 \\
$\mathrm{C}^{*}$ & $14.63^{\mathrm{b}}$ & $16.87^{\mathrm{a}}$ & 0.48 & 0.0001 \\
$\mathrm{H}^{*}$ & $33.23^{\mathrm{b}}$ & $49.28^{\mathrm{a}}$ & 1.21 & 0.0001 \\
$\mathrm{EC}(\mathrm{N})$ & $52.17^{\mathrm{a}}$ & $56.38^{\mathrm{a}}$ & 0.24 & 0.0919 \\
\hline
\end{tabular}

$\mathrm{EE}=$ error estándar; $\mathrm{EC}=$ esfuerzo al corte.

${ }^{\mathrm{a}, \mathrm{b}}$ Literales diferentes en el mismo renglón son diferentes $(P<0.05)$.

Los valores de color $\left(a^{*}, b^{*}, C^{*}\right.$ y $\left.\mathrm{H}^{*}\right)$ fueron diferentes por tratamiento. El valor de $\mathrm{L}^{*}$ no mostró diferencias $(P>0.05)$ entre castrados e inmunocastrados. Se han reportado valores de $\mathrm{L}^{*}$ similares $(P>0.05)$ en carne de machos castrados e inmunocastrados ${ }^{(10,15)}$. Lo mismo fue observado en investigaciones recientes ${ }^{(9)}$ donde registraron valores similares en valores de $L^{*}, a^{*}$ y $b^{*}$ en canales de machos castrados $\left(L^{*}=33.9 ; a^{*}=17.1 ; b^{*}=2.6\right)$ e inmunocastrados $\left(\mathrm{L}^{*}=34.0 ; \mathrm{a}^{*}=16.9 ; \mathrm{b}^{*}=2.4\right)$. A pesar de que la carne mostró un $\mathrm{pH}$ normal, los valores observados de color en este estudio son similares a los reportados de una carne DFD de una carne DFD (L*:34.8; $\left.\mathrm{a}^{*}: 18.8 ; \mathrm{b}^{*}: 6.7\right)^{(28)}$, lo que indica que los animales estuvieron expuestos a agentes estresantes previo al sacrificio.

No se observaron diferencias $(P<0.05)$ en $\mathrm{SF}$ en las canales entre machos castrados e inmunocastrados. En concordancia con criterios ya establecidos ${ }^{(29)}$, la blandura de la carne correspondió a una blandura intermedia (blanda: $22.26-35.10 \mathrm{~N}$; intermedia: $40.01-52.95 \mathrm{~N}$; dura: $57.85-70.60 \mathrm{~N})$. Lo mismo fue observado por otras investigaciones ${ }^{(10,16)}$, donde no se observaron diferencias en los valores de esfuerzo al corte en la carne de animales castrados quirúrgicamente e inmunocastrados. A su vez, no se han reportado ${ }^{(15)}$ diferencias en los valores de esfuerzo al corte debido a la condición sexual (machos castrados quirúrgicamente: $56.60 \pm 0.36 \mathrm{~N}$; immunocastrados:53.37 $\pm 0.35 \mathrm{~N}$ y machos enteros: $48.85 \pm 0.35 \mathrm{~N})$. En un estudio donde evaluaron el EC en machos, no reportaron diferencias entre castrados $(51.9 \mathrm{~N})$ e inmunocastrados $(52.9 \mathrm{~N})$ sacrificados a los 11 meses de $\operatorname{edad}^{(9)}$.

\section{Conclusiones e implicaciones}

Para fines de producción, con la castración quirúrgica a las 24 h de nacimiento en los machos Holstein, se pueden lograr animales mas pesados y con mejores características en la canal que las canales de machos inmunocastrados durante periodos de más de 200 días de engorda; 
sin embargo, es necesario evaluar el impacto del bienestar animal en becerros tras la castración.

\section{Agradecimientos}

Nuestro agradecimiento por el apoyo proporcionado para la realización de este proyecto al personal de Ganadera Mexicali S.A. y de la planta de sacrificio TIF No. 511, así como también a la MVZ. Priscila Castro Osuna por su colaboración como asesor técnico del Laboratorio Zoetis.

\section{Literatura citada:}

1. Bouissou MF, Boissy A, Neindre PL, Veissier I. The social behaviour of cattle. In: Keeling LJ, Gonyou HW editors. Social behaviour in farm animals. Oxford, UK: CABI Publishing; 2001;113-274.

2. Price EO, Adams TE, Huxsoll CC, Borgwardt RE. Aggressive behavior is reduced in bulls actively immunized against gonadotropin-releasing hormone. J Animal Sci 2003;81(2):411-415.

3. Stookey JM, Watts JM. Production practices and wellbeing: Beef cattle. Oxford. UK.: Blackwell Publishing; 2004.

4. Amatayakul-Chantler S, Jackson JA, Stegner J, King V, Rubio LMS, Howard R, Lopez $\mathrm{E}$, Walker J. Inmunocastration of Bos indicus x Brown Swiss bulls in feedlot with gonadotropin-releasing vaccine Bopriva provides improved performance and meat quality. J Anim Sci 2012;90:3718-3728.

5. Marti S, Devant M, Amatayakul-Chandler S, Jackson JA, Lopez E, Janzen ED, Schwartzkoppf-Genswein KS. Effect of anti-gonadotropin-releasing facto vaccine and band castrtion on indicators of welfare in beef cattle. J Anim Sc 2015;93:1581-1591.

6. Bonneau M, Enright W. Immunocastration in cattle and pigs. Livest Prod Sci 1995;42:193-200.

7. Mach N, Bach A, Realini C, Font-Furnols M, Velarde A, Devant M. Burdizzo prepubertal castration effects on performance, behavior, carcass characteristics, and meat quality of Holstein bulls fed high-concentrate diets. J Meat Sci 2009;81:329-334.

8. Turner AS, Mcliwraith CW. Thecniques in large animal surgery. Philadelphia, PA. Lea and Febiger; 1989. 
9. Marti S, Jackson JA, Slootmans N, Lopez E, Hodge A, Pérez-Juan M, Devant M, Amatayakul-Chantler S. Effects on performance and meat quality of Holstein bulls fed high concentrate diets without implants following immunological castration. J Meat Sci 2017;126:36-42.

10. Amatayakul-Chantler S, Hoe F, Jackson JA, Roça RDO, Stegner JE, King V, Howard R, Lopez E, Walker J. Effects on performance and carcass and meat quality attributes following immunocastration with the gonadotropin releasing factor vaccine Bopriva or surgical castration of Bos indicus bulls raised on pasture in Brazil. Meat Sci 2013;95:7884.

11. Thompson DL. Immunization against GnRH in male species (comparative aspects). J Anim Sci 2000;60:459-469.

12. Jannet F, Gerig T, Tschuor AC, Amatayakul-Chantler S, Howard R, Bollwein H, Thun R. Vaccination against gonadotropin-releasing factor (GNRF) with BOPRIVA ${ }^{\circledR}$ significantly decreases testicular development, serum testosterone levels and physical activity y pubertal Bulls. Theriogenology 2012;78:182-188.

13. Glenn CD, McMurphy CP. Feeding Holstein steers from start to finish. Vet Clin Food Anim 2007;23:281-297.

14. Ribeiro EL, Hernandez JA, Zanella EL, Shimokomaki M, Prudêncio-Ferreira SH, Youssef E, Reeves JJ. Growth and carcass characteristics of pasture fed LHRH immunocastrated, castrated and intact Bos indicus bulls. J Meat Sci 2004;68:285-290.

15. Giuliana ZM, Faria MH, Roça RO, Santos CT, Suman SP, Faitarone ABG, et al. Immunocastration improves carcass traits and beef color attributes in Nellore. J Meat Sci 2014;96:884-891.

16. Miguel GZ, Roca RO, Suman SP, Faria MH, Santos CT, Resende FD, et al. Immunocastration and surgical castration improves color attibutes of beef from Nellore males. J Meat Sci 2014;96:462-463.

17. Andreo N, Bridi AM, Soares AL, Prohmann PEF, Peres LM, Tarsitano MA, De Lima GB, Takabayashi AA. Fatty acid profile of beef from immunocastrated (BOPRIVA®) Nellore bulls. Meat Sci 2016;117:12-17.

18. García E. Modificaciones al sistema de clasificación climática de Koppen (para adaptarlo a las condiciones de la República Mexicana. México, DF. Instituto de Geografía, UNAM;1981. 
19. Hale DS, Goodson K, Savell JW. USDA beef quality and yield grades. USA: USDA; 2013.URL: http://meat.tamu.edu/beefgrading/.pdf. Consultado 10 Abr, 2017.

20. AMSA, American Meat Science Association. Meat evaluation handbook. USA. American Meat Science Association; 2001.

21. SAS. SAS software release 9.4. STAT 14.1. Cary, NC, USA: SAS Institute. Inc. 2015.

22. Steel RG, Torrie J. Bioestadistica: principios y procedimientos. México, D.F. McGrawHill Interamericana;1985.

23. Adams TE, Daley CA, Adams BM, Sakurai H. Testis function and feedlot performance of bulls actively immunized against gonadotropin-releasing hormone: Effect of age immunization. J Anim Sci 1996;71:950-954.

24. Duff GC, McMurphy CP. Feeding Holstein steers from start to finish. Veterinary Clinics of North America: Food Anim Practice 2007;23(2):281-297.

25. De Freitas VM, Leão KM, de Araujo Neto FR, Marques TC, Ferreira RM, Garcia LL F, de Oliveira EB. Effects of surgical castration, immunocastration and homeopathy on the performance, carcass characteristics and behaviour of feedlot-finished crossbred bulls. Semina: Ciências Agrárias 2015;36(3):1725-1734.

26. Mariño G, Vilca M, Ramos D. Evaluación del pH en canales de toros Holstein (Bos taurus) y Nelore (Bos indicus). Rev Investig Vet Perú 2005;16:90-95.

27. Silva JA, Patarata L, Martins C. Influence of ultimate $\mathrm{pH}$ on bovine meat tenderness during ageing. J Meat Sci 1999;52:453-459.

28. Wulf DM, Emnett RS, Leheska JM, Moeller SJ. Relationships among glycolytic potential, dark cutting (dark, firm, and dry) beef, and cooked beef palatability. J Anim Sci 2002;80:1895-1903.

29. Boleman SJ, Boleman SL, Miller RK, Taylor JF, Cross HR, Wheeler TL, Johnson DD. Consumer evaluation of beef of known categories of tenderness. J Anim Sci 1997;75:1521-1524. 